\title{
Characterization of Heat and Momentum Transfer in Sintered Metal Foams**
}

\author{
By Oliver Reutter, Jörg Sauerhering, Thomas Fend, ${ }^{*}$ Robert Pitz-Paal and Stefanie Angel
}

In this study, metal foams made by the Slip Reaction Foam Sintering (SRFS)-process are investigated concerning their thermophysical and permeability properties. Since the foam is to be applied as a functional and structural element in the effusion air cooling system of a stationary gas turbine combustion chamber, these properties are of major interest for the calculation of the temperature distribution inside the combustion chamber walls, which may be critical for the employed materials. Experimental set-ups are presented, which are used to determine permeability, the volumetric heat transfer coefficient and the effective thermal conductivity. The results are presented for a wide range of foam materials. Porosity as well as the basic metal powder and the manufacturing parameters are varied. The influence of these parameters on the measured quantities is discussed. Thermal conductivity data are determined at temperatures of up to $1200 \mathrm{~K}$. The obtained volumetric heat transfer coefficients are transferred to Nusselt-Reynolds plots, which allow generalization to the high temperature and high pressure regime. Correlations between the heat transfer properties and the permeability data are made. Using the acquired experimental data, a proposal is made for the calculation of the inner surface temperature of the combustion chamber as well as the temperature distribution inside the chamber wall, which consists of a structural element, the metal foam and a thermal barrier coating, equipped with laser drilled micro-holes.

\section{Introduction}

The foam investigated in this study is aimed to be applied as an open wall element of combustion chambers. This topic is investigated within the collaborative research center (SFB) 561 "Thermally Highly Loaded, Porous and Cooled MultiLayer Systems for Combined Cycle Power Plants". It aims at increasing the efficiency of the gas turbine by raising the gas temperature. Within this research center, the present study was carried out. Increasing the temperature leads to the necessity of actively cooling the combustion chamber walls by effusion cooling, which means that cooling air is pressed through little holes into the chamber. The metallic foam is intended to be coated with a thermal barrier layer with laser drilled boreholes and to be used as a combustion chamber wall element. ${ }^{[1]}$ A sketch of the cooling principle is shown in Figure 1. The knowledge of the temperature distribution inside the wall element (foam+coating) is important to predict the lifetime of the materials employed. To predict the temperature, flow through the wall element has to be characterized. For this, experimental data on the heat transfer char- acteristics, the thermal conductivity and the pressure drop characteristics is needed. Both, temperature prediction and determination of quantities is object of the present study.

[*] O. Reutter, J. Sauerhering, Dr. T. Fend,

Prof. Dr. R. Pitz-Paal

German Aerospace Center

Linder Hoehe

51143 Köln, Germany

E-mail: thomas.fend@dlr.de

Dr. S. Angel

University Aachen

Department of Ferrous Metallurgy

52066 Aachen, Germany

E-mail: stefanie.angel@post.rwth-aachen.de

[**] The work of the whole research center SFB561 has been funded by the German Research Foundation (DFG). This support is gratefully acknowledged. 


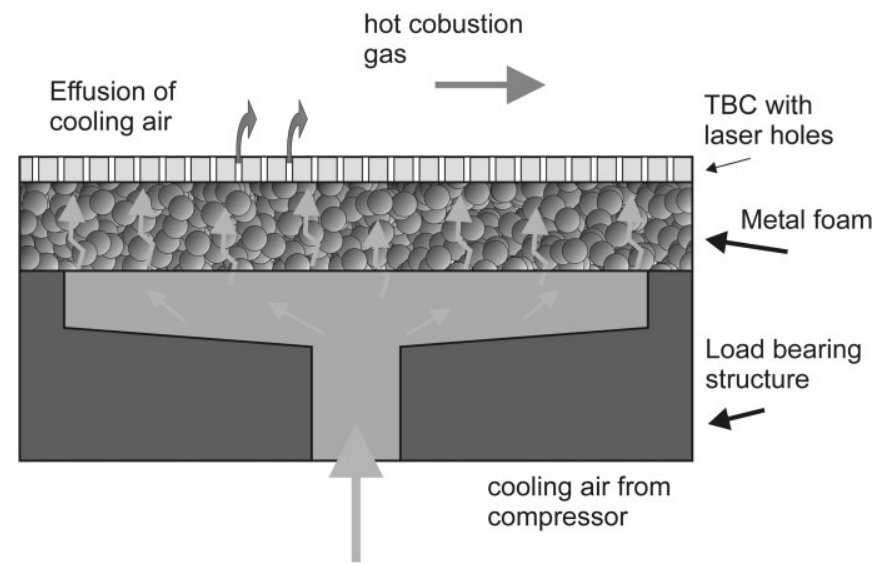

Fig. 1. Combustion chamber cooling principle.

\section{Investigated Materials}

The foams investigated in the present study are produced by the Slip Reaction Foam Sintering (SR)-process. This is a promising method to produce metallic foams with an open, porous cell structure by a chemical reaction process. As this foaming is a rather new method there is not yet ready available data concerning the pore structure or the behavior to fluid flow. The foam structure is not similar to the foams made by casting using a polymer precursor as template or by metal deposition on cellular performs like polyurethane foams. ${ }^{[2]}$ Therefore it was necessary to conduct a series of extensive experiments in order to characterize the SR foams. A first introduction and first results have been previously published. ${ }^{[3]}$ The investigated samples for this study are made of Hastelloy B-, Inconel 625and NC-powder. The grain sizes range between 50 and $150 \mu \mathrm{m}$. The iron based samples (NC) were sintered at $1170^{\circ} \mathrm{C}$, the nickel based samples (Inconel 625 and Hastelloy B) at $1200^{\circ} \mathrm{C}$. After evacuating the furnace one time before starting the sintering process, the samples were sintered under a reducing atmosphere of $100 \% \mathrm{H}_{2}$.

Samples made only of the sintered slip without foaming show a porosity of about $50 \%$ (densities of $3.8-4.2 \mathrm{~g} / \mathrm{cm}^{3}$ ) and are called sintered powder samples or matrix material. In comparison to these, the dense metals have densities of $7.87 \mathrm{~g} / \mathrm{m}^{3}$ (NC), $9.24 \mathrm{~g} / \mathrm{m}^{3}$ (Hastelloy B) and $8.44 \mathrm{~g} / \mathrm{m}^{3}$ (Inconel 625). The sintered powder samples are taken as representing the material of the pore walls in the foams. The foams have a total porosity between 62 and $87 \%$ (densities of $1.2-$ $3.7 \mathrm{~g} / \mathrm{cm}^{3}$ ). Regarding the fact, that the matrix material is porous too, it is distinguished between two types of pores. The pores can be characterized as those which arise from the hydrogen foaming, called the primary pores, and those which are the spaces between adjacent grains of the sintered powder, called the secondary pores. ${ }^{[4]}$ The secondary pores reach up to $0.3 \mathrm{~mm}$ diameter and the primary pores reach up to $3.5 \mathrm{~mm}$. All densities were determined as gravimetrical densities by weighing the foam samples and dividing this weight by the total volume of the foam sample. The total porosity was determined by using the density of the bulk material as the ref- erence. The samples were produced at the Department of Ferrous Metallurgy at Aachen University. The samples are cylinders with diameters between 64 and $81 \mathrm{~mm}$ and the length was between 9 and $30 \mathrm{~mm}$. The picture of a typical sample can be seen in Figure 2 (left) together with an optical micrograph of the sample on the right. For measurements of anisotropy effects cubical samples were used with $45 \mathrm{~mm}$ side length.

\section{Experimentally Acquired Material Data}

\subsection{Heat Conductivity}

Effective heat conductivity $\lambda_{\mathrm{EFF}}$ has been determined with the Transient Heat Source Technique also called "Hot Disk". This technique has been chosen, because the tested volume $\left(\approx 2-4 \mathrm{~cm}^{2}\right)$ is big enough to justify the assumption, that $\lambda_{\mathrm{EFF}}$ is a "volume averaged quantity", which comprises all the micro-mechanisms like conductive heat transfer in the grains, convective heat transfer in the pores and radiation heat transfer from one pore wall to another. Basics of the experimental set-up have been previously reported. ${ }^{[5]}$ The Inconel material without primary pores has been additionally measured with the Laser Flash method for comparison. It shows values of $\lambda_{\mathrm{EFF}}=0.87 \mathrm{~W} / \mathrm{mK}$ at room temperature. This is only about $1 / 10$ of the thermal conductivity of the dense metal $\left(\lambda=9.1 \mathrm{~W} / \mathrm{mK}\right.$ at $\left.25^{\circ} \mathrm{C}\right)$. The heat conductivity of the foams is even lower. As an example the results from the Inconel foams are shown in Figure 3.

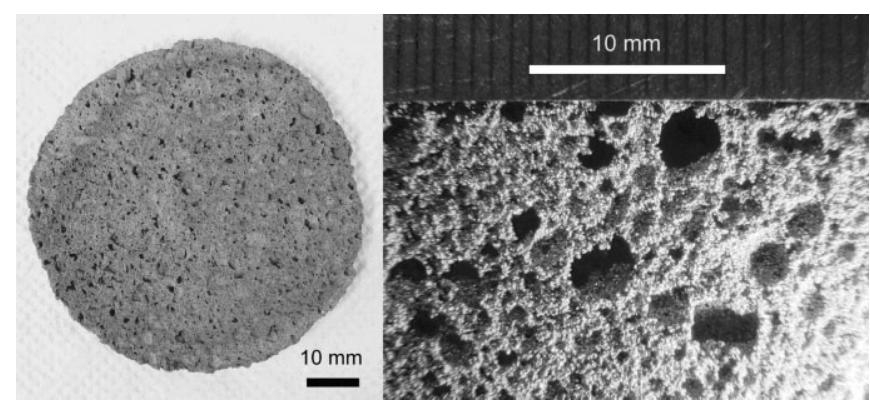

Fig. 2. Photograph of a sample of a Hastelloy B foam (left) and a detailed view of the pore structure (right).

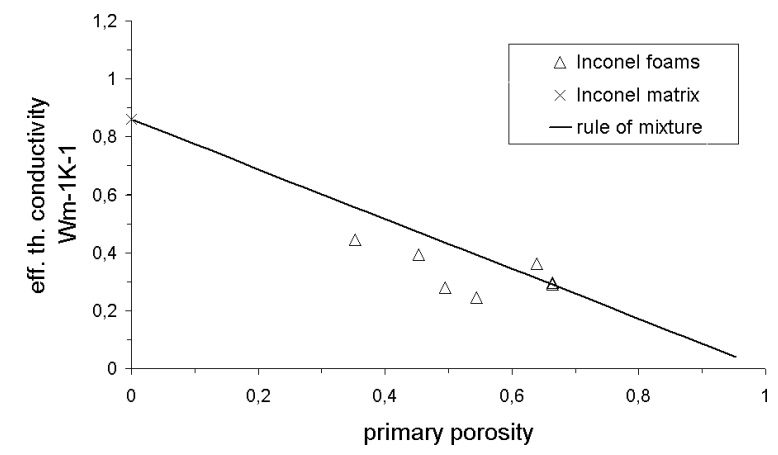

Fig. 3. Effective thermal conductivity of selected Inconel foams as a function of their total porosity. 
A simple rule of mixture holds, if only the primary porosity $\varepsilon^{*}$ is considered:

$\lambda_{\text {FOAM }}=\varepsilon^{*} \lambda_{\text {FLUID }}+\left(1-\varepsilon^{*}\right) \cdot \lambda_{\text {MATRIX }}$

The matrix thermal conductivity itself may be explained with models having been developed for packed beds. ${ }^{[6]}$

\subsection{Convective Heat Transfer Coefficients}

The effective volumetric heat transfer coefficients $a A_{V}$ (with $a$ denoting the well known convective heat transfer coefficient and $A_{V}$ the specific surface area participating in convective heat transfer usually measured in $\mathrm{m}^{2} / \mathrm{m}^{3}$ ) are also considered as volume averaged quantities describing the solid to fluid heat transfer inside the porous material. They have been determined with a transient method after Viskanta and Younis. ${ }^{[7]}$ An air flow of alternating temperature is directed through a porous sample, which causes an amplitude decrease of the temperature wave (Fig. 4). From this, $a A_{V}$ may be determined. To make use of the similarity theory, from a $A_{V}$ more general Nusselt numbers $(\mathrm{Nu})$ may be determined $\left(N u=a d / \lambda_{\text {FLUID }}\right)$. For this, a characteristic channel diameter $d$ is needed. Furthermore, the thermal conductivity of the fluid $\lambda_{\text {FLUID. }}$. For those materials, of which the results are shown in Figure 4, this diameter has been determined to approximately $0.5 \mathrm{~mm}$ by experiments observing the capillary flow of water into the pores. Reynolds numbers have been calculated from fluid flow velocity $U_{0}$ and fluid viscosity $\eta$ by $R e=d U_{0} / \eta$. The results show, that the heat transfer increases with decreasing porosity. Imagining the pore structure as a series of $\varnothing 0.5 \mathrm{~mm}$ channels, a rough approximation of the specific surface $A_{V}$ may be undertaken and Nusselt numbers may be determined directly. For the shown Hastelloy materials Nusselt numbers of $2.5<\mathrm{Nu}<5$ were calculated. These values fit well to the empirical equations for the case of laminar flow inside a tube, which give Nusselt numbers of $\mathrm{Nu} \approx 3.7$.
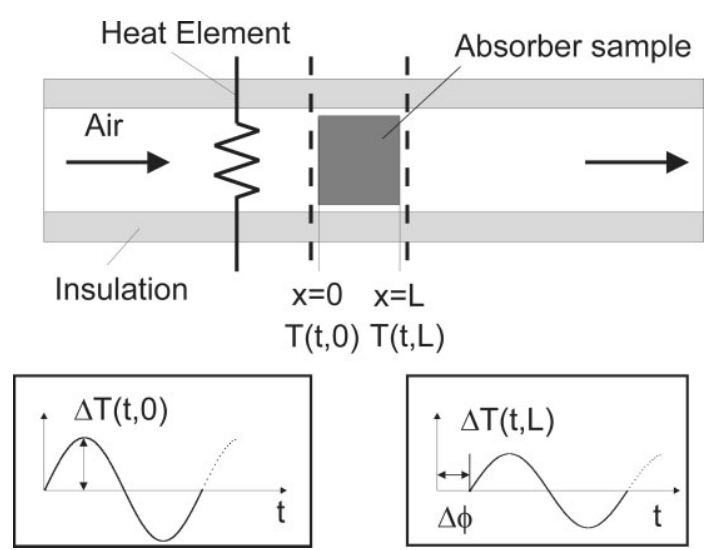

Fig. 4. Principle of the set-up used for the convective heat transfer coefficient measurements.

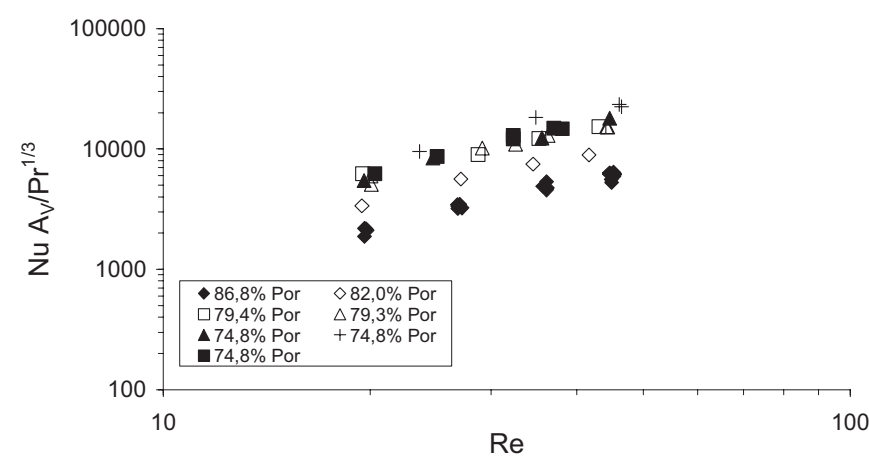

Fig. 5. Principle of the set-up used for the convective heat transfer coefficient measurements.

\subsection{Permeability}

Permeability of the foam was simply investigated by generating an air flow of various fluid velocities through the foam samples and measuring the pressure difference of the fluid before and behind the sample according to Figure 6.

Pressure loss/air velocity plots were generated as can be seen in Figure 7. The plots were fitted with the extended Darcy equation

$\frac{\Delta p}{L}=\frac{\mu_{\mathrm{DYN}}}{K_{1}} U_{0}+\frac{\rho_{F}}{K_{2}} U_{0}^{2}$

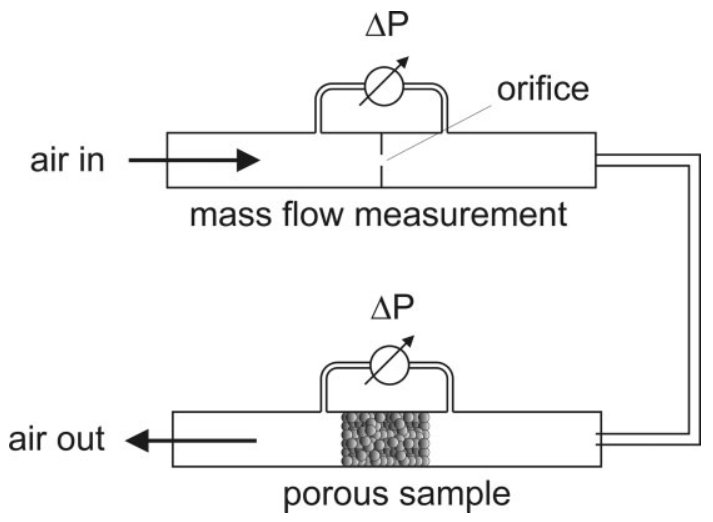

Fig. 6. Set-up used to measure permeability of foams.

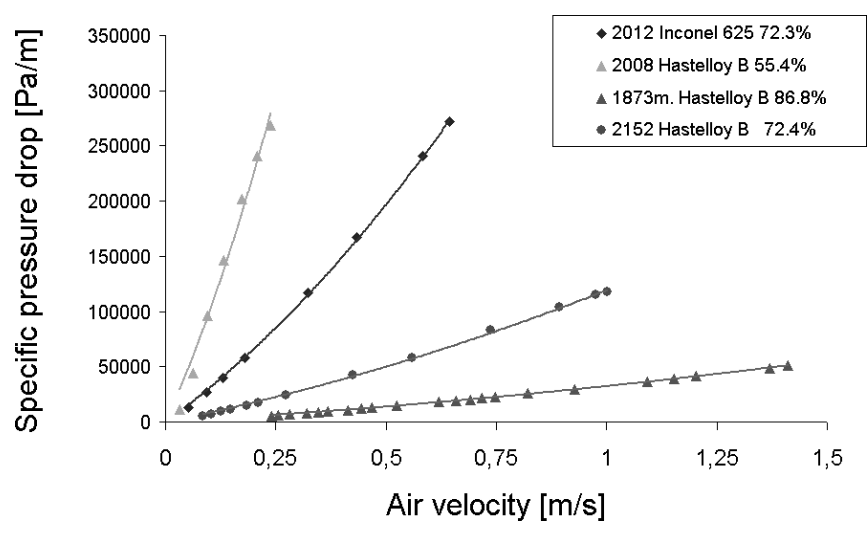

Fig. 7. Pressure loss/Air velocity plots generated during permeability measurements. 
in which $\Delta p$ denotes pressure difference, $L$ the length of the sample in the direction of flow, $\mu_{D Y N}, \rho_{F}$ and $U_{0}$ the dynamic viscosity, the density and the velocity of the fluid respectively. From the plots and the fluid data, which is taken from the literature, $\mathrm{K}_{1}$ and $\mathrm{K}_{2}$, the characteristic constants of the porous material are determined. Most frequently they are called the permeability and the inertial coefficient, because $K_{1}$ is the contribution of the viscous drag of the pores to permeability and $\mathrm{K}_{2}$ the contribution of the acceleration of the fluid particles through the tortuous path of the flow through the material. The inertial coefficients determined are shown in Figure 8 as a function of the total porosity of the foam samples. It can be clearly seen, that permeability increases significantly, when the porosity reaches approximately $70 \%$. The obvious conclusion, that the primary pores form a network of channels is not yet proved by metallographic investigations.

\section{Analytical Results}

Using the effective material data acquired in the chapters before, the heat flow balance equation for the solid and the fluid phase

$\lambda_{\text {eff }} \frac{d^{2} T_{S}}{d x^{2}}-\alpha A_{V}\left(T_{S}-T_{L}\right)=0$

$\dot{m} c_{P} \frac{d T_{L}}{d x}=-\alpha A_{v}\left(T_{S}-T_{L}\right)$

can be solved analytically as a system of differential equations. Two mass flow rates $m$ have been compared under the assumption of a constant heat flow rate

$\dot{q}=\lambda \frac{d T_{\mathrm{S}}}{d x}=1 \cdot 10^{6}\left[\frac{W}{m}\right]$

The result can be seen in Figure 9. The surface temperature of $1300 \mathrm{~K}$ (for the $\mathrm{m}=7 \mathrm{~kg} / \mathrm{m}^{2} \mathrm{~s}$ case) rapidly decreases, so that after $2 \mathrm{~mm}$ of foam already a constant temperature level is reached.

\section{Conclusions and Prospects}

The 1D-solution of the heat transfer equation is a first approach to describe the temperature distribution inside the porous combustion chamber wall. However, the presence of large pores of up to $3 \mathrm{~mm}$ diameter leads to deviations from symmetry of the temperature field and thus to a non-satisfying accuracy of the $1 \mathrm{D}$ approach. To also take into account the local effect of a primary pore on the temperature field, a $2 \mathrm{D}$ or $3 \mathrm{D}$ calculation is necessary. In this case it has to be regarded, that due to dissipation effects the effective conductivity becomes anisotrope, it becomes larger in the direction perpendicular to the main flow direction. Moreover, this effect is dependent on the velocity of the fluid flow. At the moment an experimental technique to characterize the $\lambda_{\mathrm{EFF}}$ with flow is under development.

Received: March 31, 2008

Final version: July 10, 2007

Published online: August 25, 2008

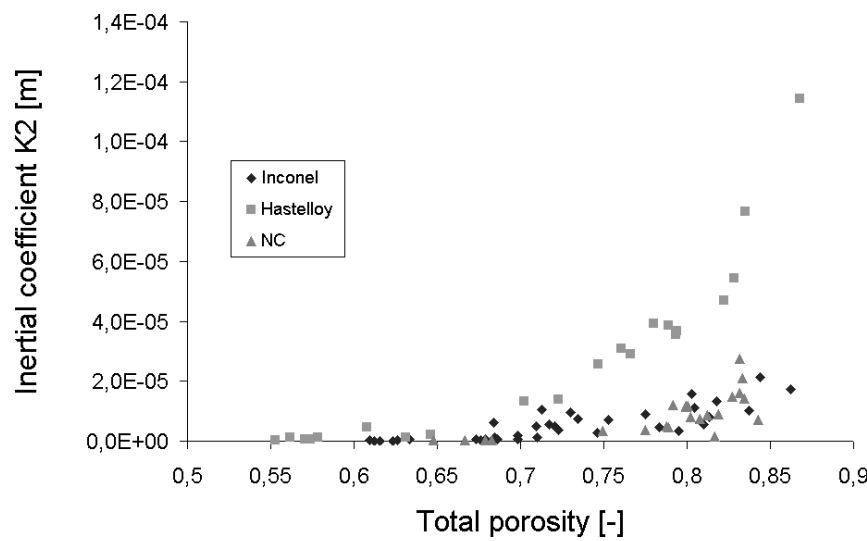

Fig. 8. The inertial coefficient as a function of the total porosity.

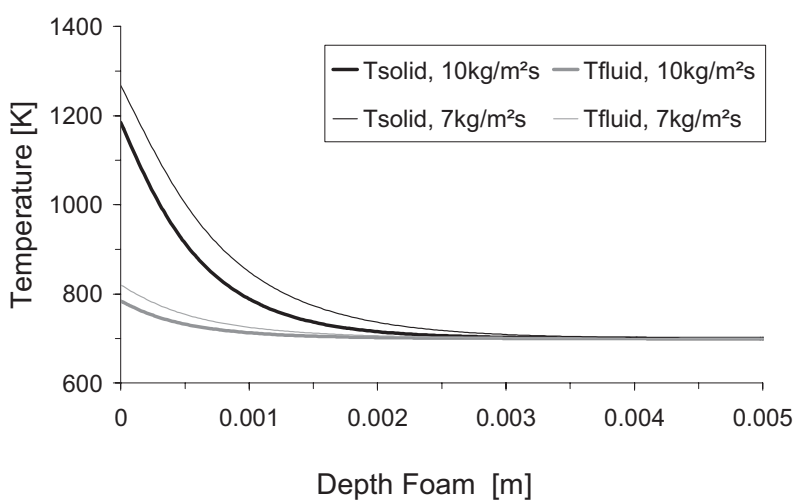

Fig. 9. Temperature distribution inside the combustion chamber wall assuming a heat flux of $50 \mathrm{MW} / \mathrm{m}^{2}$ (1D analytical solution).

[1] D. Bohn, New Mater. and Cooling Syst. for High Temp. Highly Loaded Components in Adv. Combined Cycle Power Plants, presented at the 7th Liege Conference on Materials for Advanced Power Engineering, Sept. 30 - Oct. 02, 2002

[2] M. F. Ashby, A. Evans., N. A. Fleck, L. J. Gibson, J. W. Hutchinson, H. N. G. Wadley, Met. Foams, a Design Guide, Butterworth Heinemann, 2000, 11-14.

[3] O. Reutter, J. Sauerhering, E. Smirnova, T. Fend, S. Angel, R. Pitz-Paal, Exp. Investigation of Heat Transfer and Pressure Drop in Porous Met. Foams, presented at the 4 . ASME ICNMM, June 19-21, 2006

[4] S. Angel, W. Bleck, P.-F. Scholz, Th. Fend, Steel Res. Int. 2000, 75, 483.

[5] Th. Fend, D. Trimis, R. Pitz-Paal, B. Hoffschmidt, O. Reutter, Thermal Properties Cellul. Ceram. 2008, Wiley VCH.

[6] J. Sauerhering, O. Reutter, Th. Fend, R. Pitz-Paal, S. Angel, Temp. Dependency of the Effective Thermal Conductivity of Nickel Based Met. presented at the 4. ASME ICNMM, June 19-21, 2006.

[7] R. Viskanta, L.B. Younis, Int. J. Heat Mass Trans. 1993, $36,1425$. 